\title{
Increasing the Lifetime of Wireless Sensor Networking Depending on Neighbors Relationship
}

\author{
Murad Mohammed Amin, Mohammed Hassan \\ North Technique University \\ Iraq \\ murad79@gmail.com \\ mdhmm75@yahoo.com \\ Alaa Aljomayli \\ Gazi University \\ Turkey \\ mamin@gazi.edu.tr
}

\begin{abstract}
In this Paper, Low-Energy Adaptive Clustering Hierarchy (LEACH) and K-mean clustering methods were combined into a new one. In addition, by targeting nodes that are subject to threshold value and close to each other, second level clustering was done and Energy Efficient Close Nodes Clustering (EECNC) method was developed. Aim is to use only one sensor among sensors that have same range which occur in random distribution. By applying this method, having the same data repeatedly among detection process therefore, sent packet size was reduced and the energy was saved during detection.
\end{abstract}

Keywords: EECNC, K-Mean, Clustering in WSN, WSN, K- Mean

DOI: $10.6025 / \mathrm{jes} / 2018 / 8 / 3 / 85-94$

Received: 14 March 2018, Revised 20 April 2018, Accepted 27 April 2018

(C) 2018 DLINE. All Rights Reserved

\section{Introduction}

Wireless Sensor Networks (WSNs) have extensive applications in the fields of science, environment, health, and military. Examples include plant and animal habitat monitoring, remote patient monitoring, and military defense systems. WSNs consist of hundreds or thousands of nodes, where each node has the ability to function independently. WSN nodes are small in size and need low energy. In a large geographic area, these nodes can be dispersed in a random or distributed fashion. Sensors are sometimes used in military applications, as well, such as surveillance of borders, transmitting objects or information about the enemy to other sensors and from there to the center (base station). It is therefore very important for these networks to be 
dispersed, but, at the same time, to have the ability to function in coordination with other nodes and to correct themselves in case of error [1].

Wireless Sensor Networks typically consist of many small-size sensor nodes that have limited battery life, communication bandwidth, processing speed and memory space. These sensor nodes must have different sensing features (camera, temperature measurement, sound, etc.) to monitor the environment and a central base station to transfer the sensed data. WSNs are usually deployed randomly. Thus, every node should be organized in a best and productive way. Due to the random deployment, WSN has different node densities in different regions. As a result, each node needs to consume different levels of energy to communicate with their neighbors. The lifetime of a network is quickly depleted due to both different intensities and limited energy levels. At the same time, this would lead incomplete sensing or dysfunctioning of the nodes that are thrown from an area above the ground and that have a contact with a hard floor. Therefore, it is necessary to make the nodes more durable to reduce the network error to the minimum level. Usually, the network topology changes constantly and dynamically. One of the key factors in seeking a real solution to prolong the WSN lifetime is to focus on routing methods and protocols that would possibly provide the least energy consumption [2].

The fact that battery life is limited in wireless sensor networks and that recharging the batteries of thousands of sensors from a distant source is troublesome render the energy a very crucial component for the network life. Data collection becomes a burdensome issue due to the limited energy and excess information can accumulate due to intensive use of sensor nodes. Thus, clustering sensor nodes can be considered an effective way to manage such difficulties [3].

Tree-based and clustering-based are two major communication approaches in WSN. In clustering-based communication, we should group the closest elements (nodes) of the network into the clusters and select one of these nodes as the cluster head. The cluster head manages the coordination of communication between the cluster members and the other clusters. Wireless Sensor Networks typically consist of many small-size sensor nodes that have limited battery life, communication bandwidth, processing speed and memory space. These sensor nodes must have different sensing objects (camera, temperature measurement, sound, etc.) to monitor the environment and a central base station to transfer the sensed data [4].

\section{Clustering Efforts in WSN}

Heinzelman et al. [5] developed the concept of low-energy adaptive clustering hierarchy protocol (LEACH). The techniques of LEACH includes randomized, self-configuring, adaptive to clustering, localized control for data transfers, low-energy media access, and data processing. The LEACH protocol has many cycles but takes place in two stages: Set-up phase and steadystate phase. During the set-up phase, clusters are formed in an adaptive manner, whereas in the steady-state phase the transfer of the data takes place. LEACH uses TDMA or Code Division Multiple Access (CDMA) MAC to reduce inter-cluster and intracluster collisions. Cluster formation is based on many properties such as the number of sensor types, communication coverage, and geographical location. The energy consumption caused by the information aggregation from the sensor node to reach the receiver depends on the number of cluster heads and the radio signal coverage of different algorithms. Because energy consumption can be reduced by organizing the sensor nodes in the clusters.

Younis et al. [6] suggested a distributed clustering method by the name of Hybrid Energy-Efficient Distributed Clustering(HEED), which targets transmission power. This method aims to prolong the network lifetime by distributing energy consumption and terminating the clustering process within a fixed number of iterations. They produced uniformly distributed cluster leaders and small-size clusters by minimizing the control overrun of the algorithms. HEED assumes that energy consumption is not uniform among the nodes and that all nodes have equal importance.

Lindsey et al. [7,8] developed an energy efficiency-oriented aggregation by the name of PEGASIS in sensor information systems. As compared to the LEACH algorithm, PEGASIS is a chain-based protocol that provides more improvement. Within PEGASIS, each node only communicates with its immediate neighbors and directs it to transmit to the base station. Thus, the energy consumed for each cycle is reduced. Using the Greedy Approach, the nodes are organized to form a chain (as seen in Figure 1). The base station can then calculate this chain and send it to the sensor nodes. Energy saving in PEGASIS comes at various stages compared to LEACH. First, the local data collection, the distance that most sensor nodes transmit, and the distance that most circuit nodes transmit are far less than the distance that head cluster transmits in LEACH [9]. Second, only one node sends the communication to the base station in each communication cycle. PEGASIS performs well by limiting the number of shipment and eliminating the dynamic additional loads.

86 Journal of Electronic Systems Volume 8 Number 3 September 2018




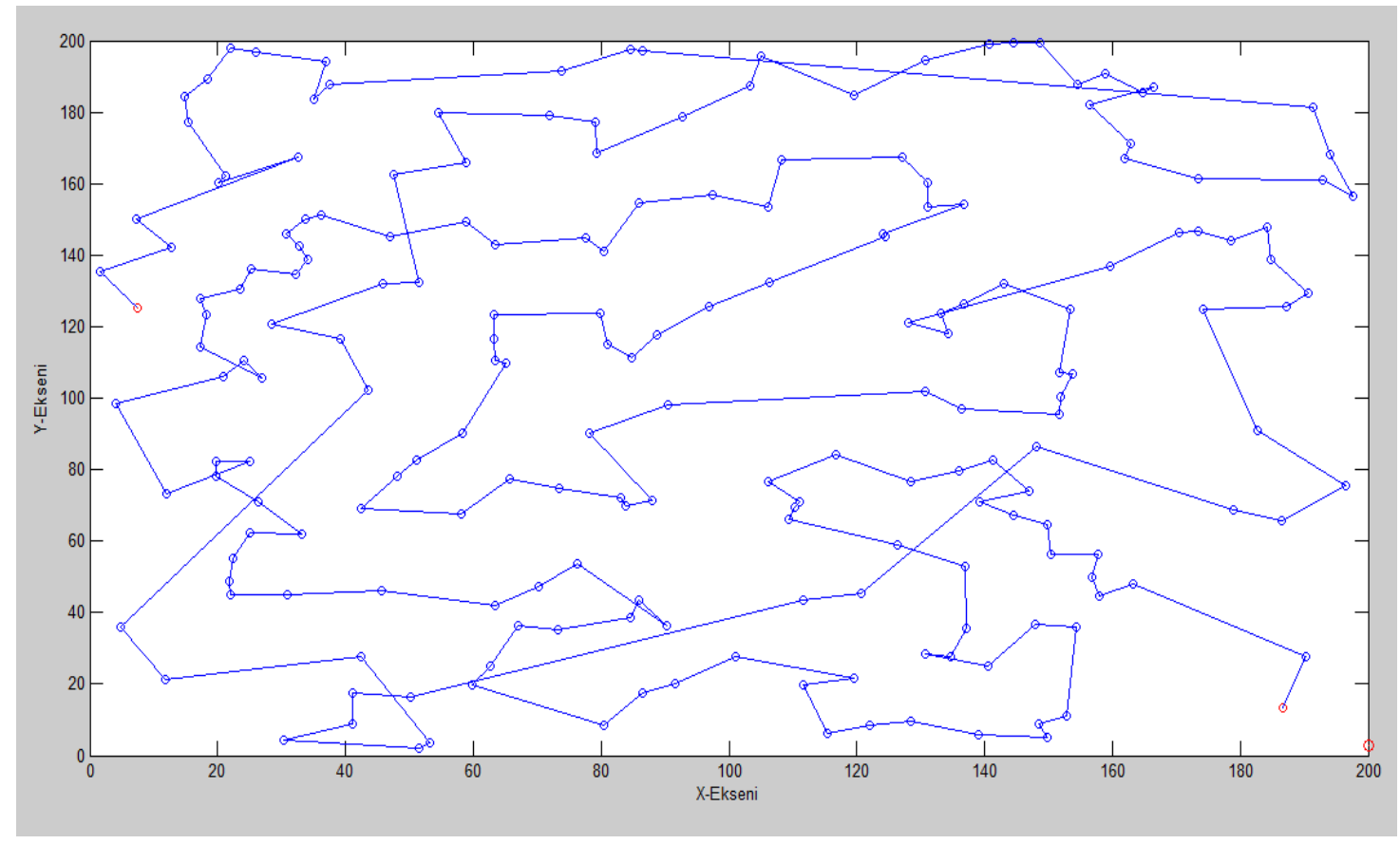

Figure 1. The cluster formation of PEGASIS algorithm in the simulation program environment

\section{Enhancement of Energy Efficiency using WSN Cluster Algorithms}

It is now possible to generate sensor nodes that perform sensing and transmitting functions. However, some applications require hundreds or thousands of sensor nodes and these nodes needs to use their limited disposable energies quite attentively. Undoubtedly, it is important to design efficient sensor circuits. However, developing a good routing technique at that point is an equally important issue in terms of extending network life. The direct transmission has been examined first to overcome constraints. In such a method, the sensor node senses the environment and transmits the data directly to the base station. Although the method specified provides data security, it also leads to excessive power consumption. (Especially nodes that are far from the base station) die early due to the energy spent for transmission while they are supposed to do sensing. Consequently, they leave the field abandoned [10].

The minimum transmission power technique should be used to solve the distance problem. In this technique, multi-hop transmission is used instead of direct transmission. Particularly, the nodes that are far from the base station transmit the data to the nearby stations and they transmit them to the base station so that the energy distribution is provided and the network lifetime is extended [11]. However, one of the best-proven methods to save energy in routing is clustering [12]. Accordingly, when nodes are distributed, they organize themselves and select a node as the cluster head. The task of cluster head is to aggregate the data from the other nodes in the cluster and transfer them to the base station while other nodes perform sensing. Thus, the division of labor optimizes energy loss and extends network lifetime. There are many clustering-based studies conducted in recent years. Although the cluster head selection mechanism differs between the methods, the main purpose of energy saving remains the same [13].

LEACH, PEGASIS, TEEN, APTEEN, and HEED are the most commonly used clustering algorithms. LEACH algorithm consists of three stages: signal propagation (position specification), cluster formation and sensing.

\section{Problem Definition and Work Performed: EECNC}

The main purpose of this study is to prevent multiple sensor nodes from sensing and generating same data due to random deployment and having the same communication distance. It is known that sensor nodes carry more than one property at the same time (e.g. heat sensation, sound, image, etc.). Some of the sensor nodes are concentrated in the same vicinity after they are 
thrown into the areas that have a difficult access such as the volcanoes and the battlefield. Proximity can vary between different applications. For example, the distance for the cameras that sense a moving object differs from the distance for the sensors that collect data on temperature should be different. Clustering sensor nodes is an effective way to overcome such problems. Thus, the determination of proximity between different applications is performed under the control of the user. The objectives are to operate a single sensor that operates in the same area, to prevent transmitting the same information and consequently to achieve energy saving. Figure 2 shows the accumulation of excess data that describes the sensing area indentations and leading to energy loss.

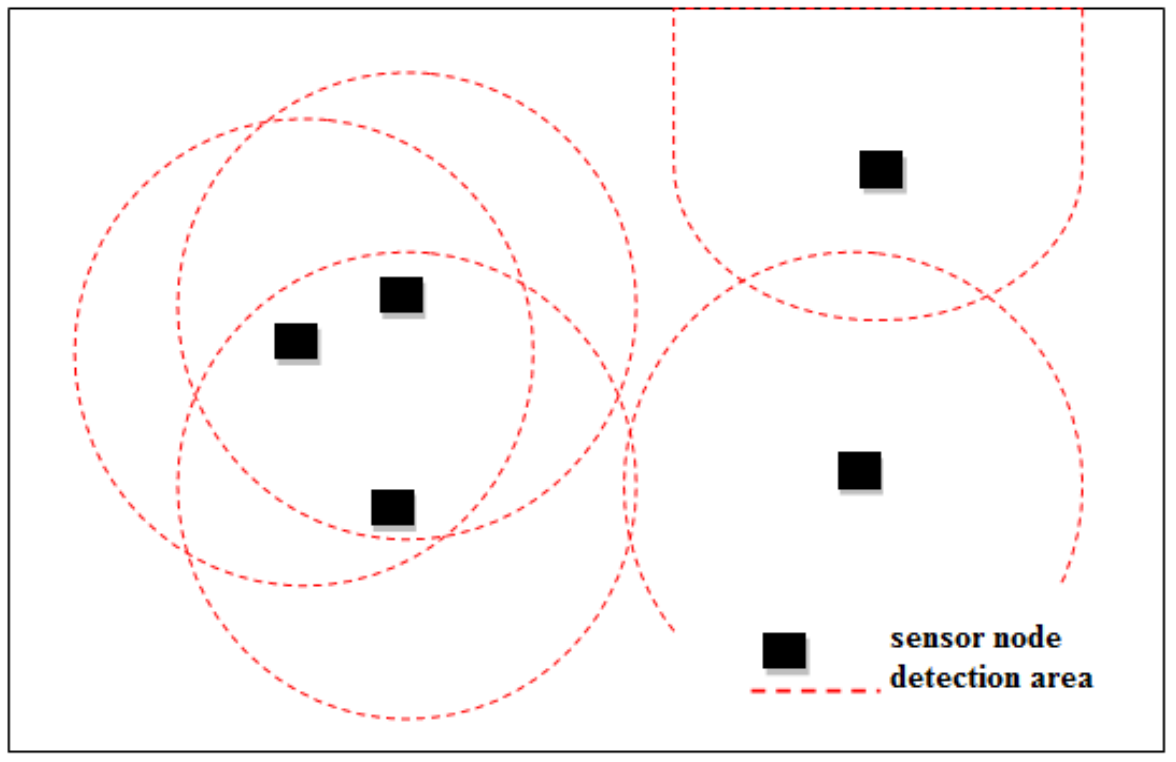

Figure 2. Nodes in the same communication range due to random deployment

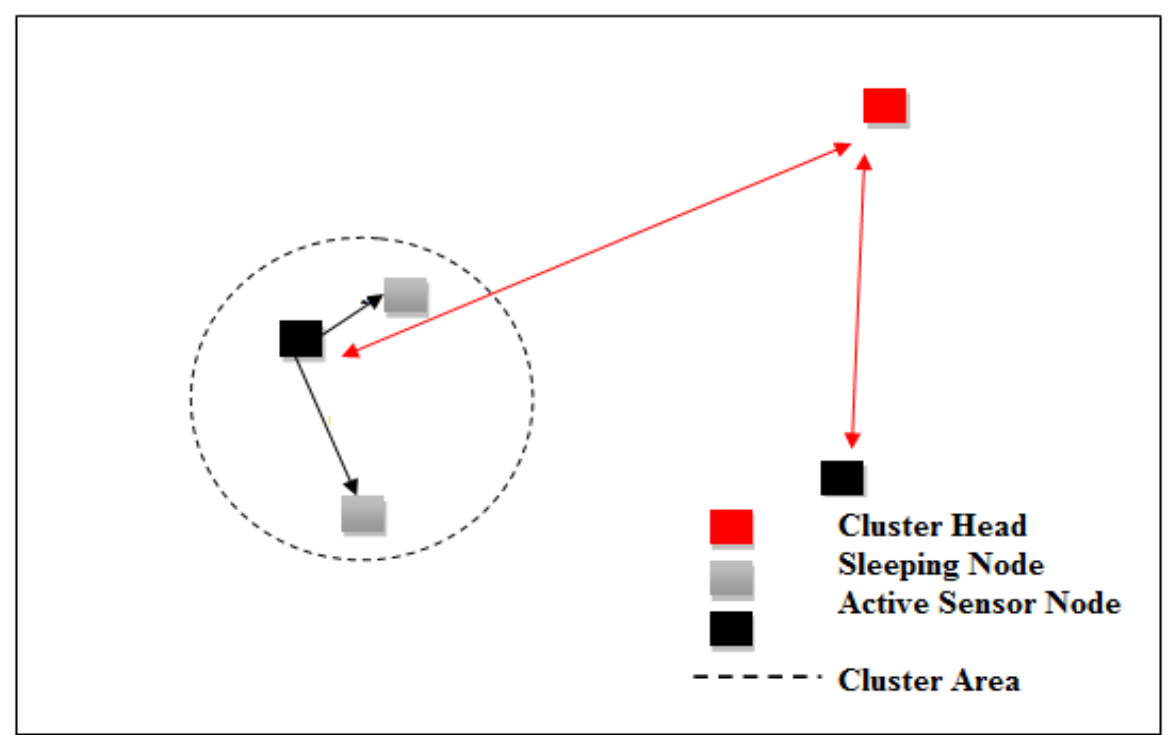

Figure 3. Nodes in the second stage cluster that keeps proximity range large

Cluster structure architecture is used to accomplish this objective. This study is conducted on LEACH cluster structure. While the nodes in the cluster formed the original cluster in the first stage, the adjacent nodes that are determined based on their location organize themselves to form the second stage cluster.

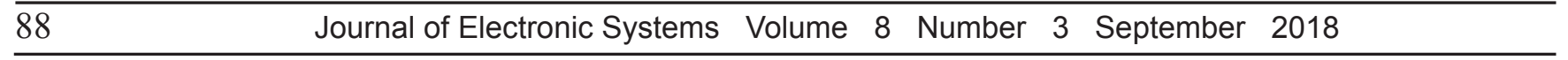


The only difference between the second stage cluster and normal clusters is that only cluster head performs the sensing and transmitting jobs and actively operates. Other nodes are in the sleeping mode. When cluster head reaches a certain threshold value, it sends other nodes a command to become active and goes to the sleep mode itself as a cluster head. Figure 3 show the cluster elements and active nodes according to the communication range.

In this study, it is aimed to extend the network life by using a random distribution of nodes in wireless sensor networks and using K-Mean clustering architecture to obtain higher energy efficiency. In the proposed study, the nodes that are located close to each other are prevented from operating at the same time using a threshold value. The clustering process consists of two stages. In the first stage, randomly distributed nodes are transformed into clusters by means of the K-Mean algorithm. Thus, data transfer from the nodes to the base is achieved with less energy loss. In the second stage, a cluster head was determined by using the neighborhood table created in the first stage and by setting up a communication between the adjacent nodes. The cluster head will start active sensing and put other nodes in the cluster into the sleep mode. The active node will command another node to be the cluster head after losing its energy and will go to the sleep mode. This will extend the network lifetime and allow different sensing areas to be specified according to different sensing needs.

\section{Proposed Study}

The work suggested in this study is based on the following steps:

1. Distributing the WSN nodes to start the parameters.

2. Use a network topology to measure the value.

3. Performing cluster heads selection.

4. Sending packets between nodes to start communication.

5. Calculating the results (e.g. network life, number of dead nodes, etc.) for EECNC method.

6. Calculating the results for other methods (HEED, PEGASIS, TEEN, APTEEN, and LEACH).

7. Evaluate performance of between methods and compare the results.

\subsection{Assumptions}

The simulation assumes that WSN nodes are distributed randomly, and wireless networks have the following characteristics:

1. Always have data to send.

2. Only one base station located in the middle of the network is available.

3. If the remaining energy of the node is not sufficient to send packets to the cluster head, it is considered dead.

4. Signal interference and loss are neglected.

5. Lost energy for communication depends on the distance between node and cluster head.

\subsection{Simulation Result}

WSN distribution and comparison is not easy in this part of the simulation because there are too many parameters to consider such as energy parameters, cluster head selection parameters, and transmission parameters. MATLAB platform is used to form different clusters according to identified methods and to compute life cycle.

\subsection{Parameters}

Each parameter included in the simulation is crucial for extending the life of wireless sensor network. The definition of the parameters is as follows:

1. Packet Delivery Rate: The ratio of the number of packets sent from the source node to the number of packets arriving at the destination node. A value close to 1 indicates higher performance.

2. Network Life: The sum of the time since the network deployment until the last sensor node has run out of power and no data 
is available to the base station.

3. Productivity: One of the most important factors for performance measurement in network analysis. Correcting the incorrect packet delivery instead of resending the packet.

\subsection{Energy Efficient Close Nodes Clustering Implementation}

After specifying the number of nodes, the program will ask for the cluster area because the number of nodes in the cluster increases or decreases according to space. The program asks the proximity at the last stage. What is important at this point is to determine the purpose the distance sensor used for. Figure 4 shows the cluster elements and the central cluster head specifying the cluster field 50 units.

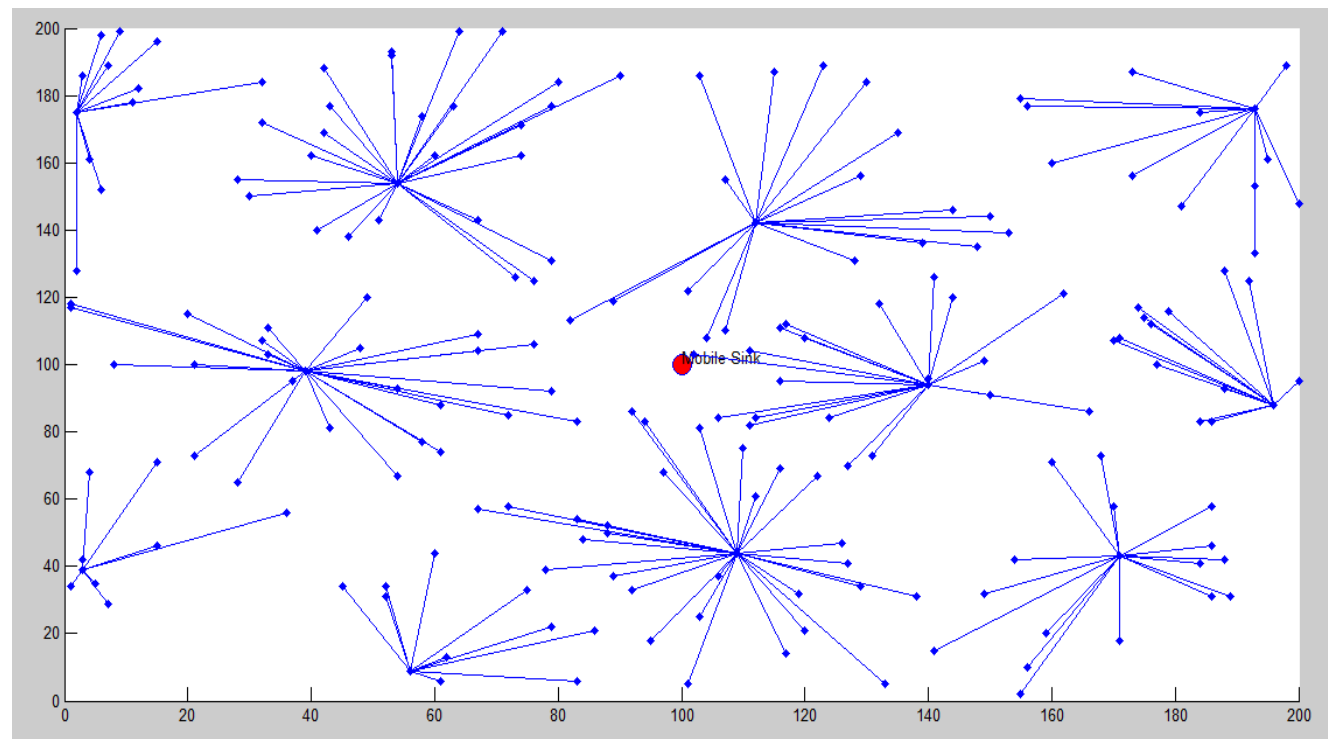

Figure 4. Cluster specifying the cluster area with a radius of $50 \mathrm{~m}$

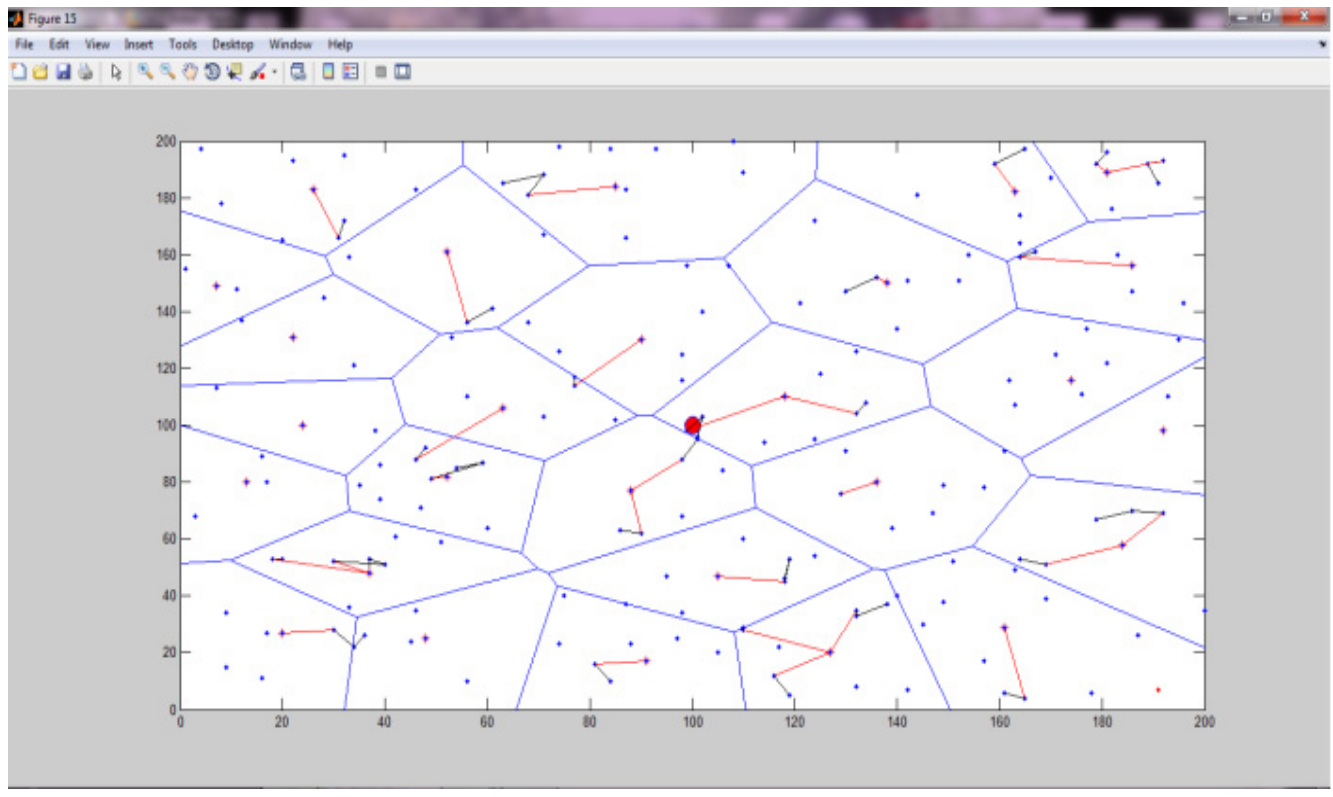

Figure 5. Two-stage clustering illustration 
When the program starts to work, only the cluster heads of the second stage clusters and the nodes determined according to the proximity are running. As shown in Figure 5 the red lines indicate active nodes, while the black lines indicate sleeping nodes. As seen in Figure 5, nodes that only achieve the proximity form a second stage cluster (nodes connected to each with a black line form the cluster) and perform like single node. The red line indicates that the active node is communicating with the cluster head.

In order to compare the EECNC method to other clustering methods, the program performance was first measured. The application will wait for the distance to be determined to form a cluster after the number of nodes entered and the nodes are dispersed randomly in the cluster space. The determination of distance will, in turn, facilitate the determination of the number of clusters and the creation of the dynamic cluster. To form the second stage clustering the proximity will be required. Proximity will determine the number of nodes for each cluster. Each cluster is composed of 1,2 or more nodes, but in each cluster, only one node operates at a time. The decision about the operating node is dependent on the energy level. The node with the highest energy level will be active. At the end of each iteration, the application controls whether the energy level of the active node has come to the critical point. When the energy level is too low, another node in the cluster that is in the sleep mode will be active and the old cluster head will go to sleep mode. The application detects the nodes that run out of energy (dead nodes) and add them to the counter for performance measurement. The number of dead nodes and the level of energy loss is determined in four steps at the end of each $(25,50,75$, and 100 steps).

For each method (EECNC, PEGASIS, HEED, TEEN, and LEACH) the common parameters specified in Table1. The following code shows the assignment of parameters.

\begin{tabular}{|l|l|}
\hline Parameters & Value \\
\hline Number of sensor nodes & 200 count \\
\hline Cluster area & $25 \mathrm{~m}$ \\
\hline Proximity & $5 \mathrm{~m}$ \\
\hline Area & $200 \mathrm{X} 200 \mathrm{~m}^{2}$ \\
\hline Base station coordination & $100 \mathrm{X} 100 \mathrm{~m}^{2}$ \\
\hline Energy spent on transmitting and receiving per bit & $500 \mathrm{~nJ} / \mathrm{bit}$ \\
\hline Iteration & 100 \\
\hline Energy for distribution & $10 * 0.000000000001 \mathrm{~J}$ \\
\hline Transmission energy & $50 * 0.000000000001 \mathrm{~J}$ \\
\hline Reception energy & $50 * 0.000000000001 \mathrm{~J}$ \\
\hline Energy for data collection & $5 * 0.000000000001 \mathrm{~J}$ \\
\hline
\end{tabular}

Table1. Values used for 100 iterations

The results of comparison reveal that EECNC and PEGASIS show the highest performance. The LEACH algorithm is a major energy loss when used in its own form. Although the algorithm application is useful in general terms, it seems that it is not useful in terms of cost.

In order to improve the performance of the EECN method, the comparison between EECNC and LEACH, HEED, TEEN, APTEEN, and PEGASIS algorithms with less number of nodes and more energy levels was repeated in accordance with the parameters shown in Table 2 and the Results for the energy level shown in Figure 6.

Finally, EECNC showed the highest performance once again as a result of simulation with a random distribution of 200 nodes with the same algorithms. Although it continues to sense with fewer nodes, it is seen that network life in EECNC will be longer than those of other algorithms. Table 3 shows the parameters and the results from the simulation Figure 7. 


\begin{tabular}{|l|l|}
\hline Parameters & Value \\
\hline Number of sensor nodes & $100 \mathrm{count}$ \\
\hline Cluster area & $35 \mathrm{~m}$ \\
\hline Proximity & $7 \mathrm{~m}$ \\
\hline Area & $100 \mathrm{X} 100 \mathrm{~m}^{2}$ \\
\hline Base station coordination & $50 \mathrm{X} 50$ \\
\hline Energy spent on transmitting and receiving per bit & $50 \mathrm{~nJ} / \mathrm{bit}$ \\
\hline Iteration & 12000 iterations \\
\hline Energy for distribution & $10 * 0.0000000000001 \mathrm{~J}$ \\
\hline Transmission energy & $50 * 0.000000000001 \mathrm{~J}$ \\
\hline Reception energy & $50 * 0.0000000000001 \mathrm{~J}$ \\
\hline Energy for data collection & $5 * 0.000000000001 \mathrm{~J}$ \\
\hline
\end{tabular}

Table 2. The second stage comparison parameters

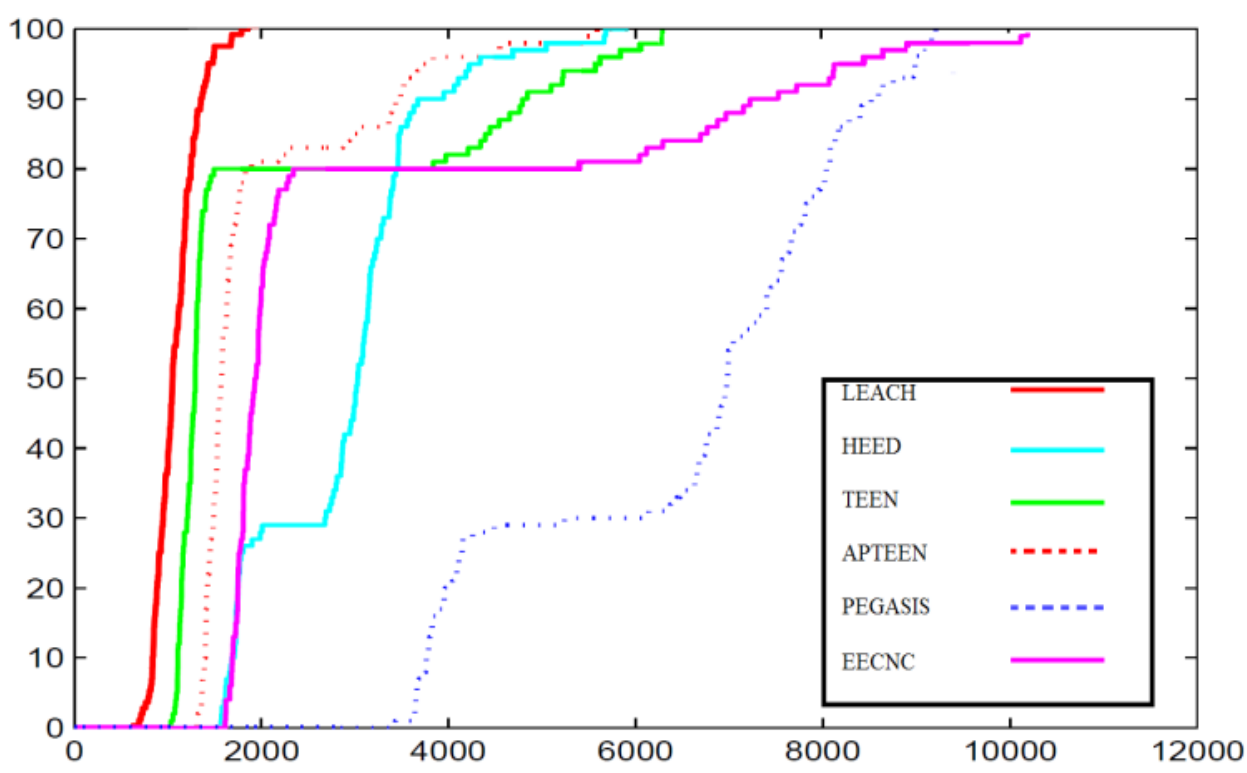

Figure 6.Comparison result according to energy level between methods

\section{Conclusions and Recommendations}

The purpose of this study is to achieve energy saving in wireless sensor networks by preventing sensor nodes from multiple sensing and to control communication range for different applications. To extend or shorten the communication range (e.g. the range of the nodes that are thrown into a forest to sense temperature should be longer than the range for sensing moving objects) to collect data in a more efficient way, to prevent sensor nodes from sensing the same objects or conditions are all important considerations to reduce energy loss.

In this study, a higher energy efficiency is achieved in wireless sensor networks by utilizing clustering architecture method as compared to a setting where sensor nodes are randomly distributed. In the experiment, sensor nodes that are deployed close to each other are prevented from operating at the same time using a threshold value. For simulation, MATLAB program is used.

\begin{tabular}{llllll}
\hline 92 & Journal of Electronic Systems & Volume 8 & Number 3 & September 2018 \\
\hline
\end{tabular}




\begin{tabular}{|l|l|}
\hline Parameters & Value \\
\hline Number of sensor nodes & $200 \mathrm{count}$ \\
\hline Area & $200 \mathrm{X} 200 \mathrm{~m}^{2}$ \\
\hline Base station coordination & $100 \mathrm{X} 100$ \\
\hline Energy spent on transmitting and receiving per bit & $50 \mathrm{~nJ} / \mathrm{bit}$ \\
\hline Number of bits per packet & $4000 \mathrm{bit}$ \\
\hline Energy for distribution & $10 * 0.000000000001 \mathrm{~J}$ \\
\hline Transmission energy & $50 * 0.000000000001 \mathrm{~J}$ \\
\hline Reception energy & $50 * 0.000000000001 \mathrm{~J}$ \\
\hline Energy for data collection & $5 * 0.000000000001 \mathrm{~J}$ \\
\hline
\end{tabular}

Table 3. Parameters applied to the methods for 200 nodes

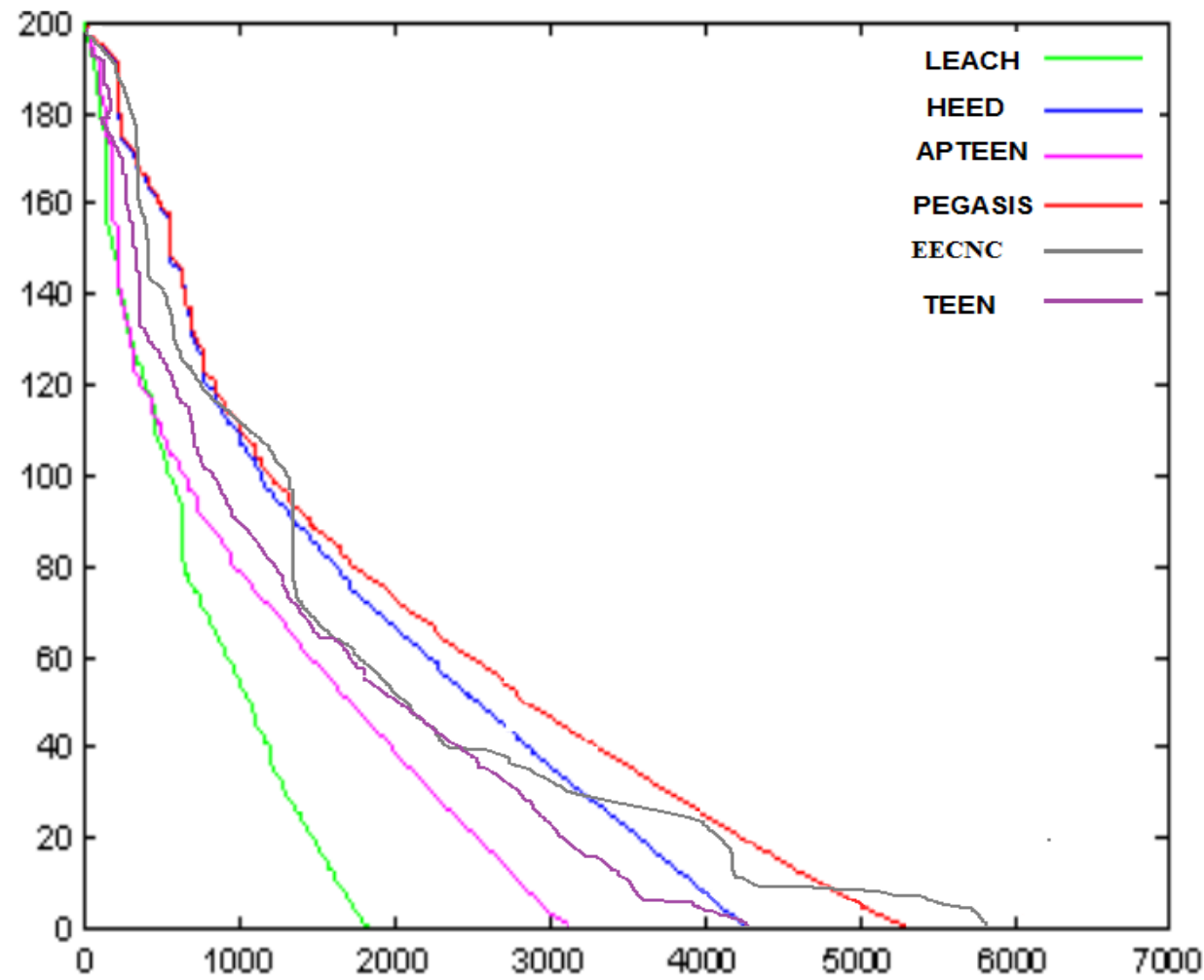

Figure 7. Comparison results according to energy levels for 200 nodes between methods

In this study, it is aimed to extend the network life by using random distribution of nodes in wireless sensor networks and using $K$-Mean clustering architecture to obtain higher energy efficiency. In this study, the researcher aims to obtain a higher energy efficiency in wireless sensor network where sensor nodes are randomly distributed by utilizing $K$-mean clustering architecture. In the proposed study, sensor nodes that are deployed close to each other are prevented from operating at the same time using a threshold value. The clustering process consists of two stages.

In the first stage, randomly distributed nodes are transformed into clusters by means of the $K$-Mean algorithm. Thus, data transfer from the nodes to the base is achieved with less energy loss. 
In the second stage, using the neighborhood table which is created during the first sage and setting up a communication between closely located nodes, a cluster head is determined. When the cluster head is activated, other nodes in the cluster are put into the sleep mode. The active node will lose its energy after a while and then it will command another node to be active as a cluster head before it goes into sleep mode. This will extend the lifetime of the network and allow different sensing areas to be specified according to different sensing needs.

It is clear from the study that the simulated method has better energy saving than the other clustering methods and extends the network life even more. In the majority of past studies aiming at energy saving in the field of WSN, energy saving is tried based solely on clustering regardless of differences between applications.

In addition to the findings, some recommendations are presented below for future studies:

Most studies on wireless sensor networks' energy saving via routing protocols usually focus on data collection and transmission of information to the base. However, energy saving during communication is rarely studied.

The current study focuses on prolonging the network life. It is possible to extend the findings of this study and improve it by focusing on the quality of service.

\section{References}

[1] Al Nuaimi, M., Shuaib, K., Al Nuaimi, K. (2014). Clustering in WSN Using Node Ranking with Hybrid Nodes Duty-Cycle and Energy Threshold. IEEE 13th International Symposium on Network Computing and Applications, Cambridge, MA. (21-23 August)

[2] Nithyakalyani, S., Gopinath, B. (2015). Analysis of Node Clustering Algorithms on Data Aggregation in Wireless Sensor Network. Journal of Scientific \& Industrial Research, 5(72), 38-42.

[3] Kulik, J., Heinzelman, W., Balakrishnan, H. (2002). Negotiation-based protocols for disseminating information in wireless sensor Networks. Wireless Networks Journal, 8(2), 169-185.

[4] Megerian, S., Hao, C. (2006). Cluster sizing and head selection for efficient data aggregation and routing in sensor networks. IEEE on Wireless Communications and Networking Conference, Las Vegas, NV. (3-6 April).

[5] Yuhui, J., Junping, H. (2008). A Time- based Cluster - Head Selection Algorithm for LEACH. IEEE Symposium on Computers and Communications, Marrakech. (6-9 Haziran)

[6] Younis, O., Fahmy, S. (2004). HEED: A Hybrid, Energy-Efficient, Distributed Clustering Approach for Ad Hoc Sensor Networks. IEEE Transactions on Mobile Computing, 3(4), 366-379.

[7] Lindsey, S., Raghavendra, C. S. (2002). PEGASIS: Power-Efficient Gathering in Sensor Information Systems. IEEE Aerospace Conference Proceedings. 3(3)1125-1130.

[8] Yun, Z., Chang-RI, L., Zibo, Z. (2010). A clustering algorithm based on cell combination for wireless sensor networks. 2010 Second International Workshop on Education Technology and Computer Science, Wuhan. (6-7 March).

[9] Guozhi, L., Wang, R., Zheng, C. (2007). A clustering algorithm based on vi rtual area partition for heterogeneous wireless sensor networks. IEEE International Conference on Mechatronics and Automation, Harbin. (5-8 August)

[10] Prakash, R., Amis, A. D., Yuong, T. (2011) Max - min d -cluster formation in wireless ad hoc networks. Nineteenth IEEE Annual Joint Proceeding on Computer and Communication Societies, 9(2), 112-119.

[11] Barcelo-ordinas, J. M., Alaei, M. (2010). Clustering based on overlapping FoVs for wireless multimedia sensor networks. IEEE Wireless Communication and Networking, Sydney, NSW. (18-21 April).

[12] Youssef, M., Youssef, A. (2014). Overlapping multi -hop clustering for wireless sensor networks. IEEE transactions on Parallel and Distributed Systems, 5(1), 1-15.

[13] Pratyay K., Prasanta, K. (2014). A novel differential evolution based clustering algorithm for wireless sensor Networks. Applied Soft Computing, 25(1), 414-425.

$94 \quad$ Journal of Electronic Systems Volume 8 Number 3 September 2018 